\title{
Characterisation of a clinical isolated Aspergillus lentulus strain using a Galleria mellonella infection model
}

\author{
Li-Juan Zhang, Xiao-Dong Wang, Ming-Shuo Ji, Hadiliya Hasimu, Paride Abliz \\ Department of Dermatology, The First Affiliated Hospital of Xinjiang Medical University, Urumqi, China \\ Contributions: (I) Conception and design: LJ Zhang, P Abliz; (II) Administrative support: P Abliz; (III) Provision of study materials or patients: MS Ji, \\ H Hasimu; (IV) Collection and assembly of data: LJ Zhang, XD Wang; (V) Data analysis and interpretation: LJ Zhang, XD Wang; (VI) Manuscript \\ writing: All authors; (VII) Final approval of manuscript: All authors. \\ Correspondence to: Paride Abliz. Department of Dermatology, The First Affiliated Hospital of Xinjiang Medical University, 137 South Carp Hill Road, \\ Xinshi, Urumqi 830054, China. Email: palida_abu@163.com.
}

Background: In recent years, the number of invasive aspergillus infection cases caused by Aspergillus lentulus (A. lentulus) has gradually increased and this fungus is usually difficult to distinguish from Aspergillus fumigatus in morphology. All of these presents a great challenge to the treatment of invasive fungal infections caused by $A$. lentulus. The present study aims to discuss the antifungal resistance, virulence and inflammatory factors' changes after the infection of larvae of $A$. lentulus separated from patients with chronic obstructive pulmonary disease (COPD) to reflect the host immune response.

Methods: A. lentulus isolated from COPD patients was identified by morphology and molecular biology, and its drug sensitivity was determined in vitro. Then the virulence factors and inflammatory response related factors of $A$. lentulus were determined by the model of $A$. lentulus infecting larvae. These were divided into three groups: A. lentulus standard strain, A. lentulus strain isolated from patients; PBS control. The infection model was formed by injecting the suspension of $A$. lentulus at a concentration of $1 \times 10^{6} \mathrm{CFU}$ into larvae, in order to determine the (1,3)- $\beta$-D-glucan and galactomannan levels, and determine the caspase- 1 and TNF- $\alpha$ concentration in Galleria mellonella larvae by RT-PCR.

Results: The results revealed that $A$. lentulus had good sensitivity to itraconazole, voriconazole and micafungin, while $(1,3)-\beta$-D-glucan was negative in the two groups. The level of galactomannan in the two groups was higher than that in the control group, and the difference was statistically significant $(\mathrm{P}<0.05)$. However, there was no statistical difference between the standard strain group and patient strain group $(\mathrm{P}>0.05)$. After the infection of larvae, caspase-1 and TNF- $\alpha$ in the Galleria mellonella larvae increased in the two groups, and these elevated levels were statistically significant in both groups $(\mathrm{P}<0.05)$. However, there was no significant difference between the two groups $(\mathrm{P}>0.05)$.

Conclusions: There is no significant difference in virulence factor and host inflammatory response between $A$. lentulus isolated from COPD patients and standard strains. Galactomannan has more advantages in the early detection of $A$. lentulus invasive infection. Furthermore, the caspase-1-mediated inflammasome pathway may be involved in the host immune response to $A$. lentulus.

Keywords: Chronic obstructive pulmonary disease (COPD); Aspergillus lentulus (A. lentulus); larvae; caspase 1; TNF alpha

Submitted Feb 20, 2020. Accepted for publication Nov 03, 2020.

doi: $10.21037 /$ jtd-20-961

View this article at: http://dx.doi.org/10.21037/jtd-20-961 


\section{Introduction}

At present, Aspergillus fumigatus has become the most common air disseminated pathogenic fungus, which can cause serious fatal invasive infection in patients with immune impairment (1). Aspergillus lentulus (A. lentulus) was isolated and identified by Balajee in the secretions of four patients who underwent hematopoietic stem cell transplantation in 2005, and this became a new record aspergillus as a sister fungus of Aspergillus fumigatus (2). For the first time in 2008, Alhambra et al. (3) reported that this fungus could cause invasive infections in non-neutropenia patients. In 2011, a new species of $A$. lentulus that belongs to Aspergillus and Aspergillus fumigatus was isolated from sputum samples of an elderly male patient with chronic cor pulmonale complicated with acute pulmonary infection in 2011. The in vitro antifungal susceptibility test revealed the resistance to multiple antifungal agents. The patient died due to aggressive lung fungal infection after active treatment. This was the first case of $A$. lentulus infection and non-immunodeficiency in China. The clinical history of this patient's infection suggest that the fungus can cause fatal consequences, not only to patients with impaired immune function, but also to patients with non-immune impairment. In recent years, the number of invasive Aspergillus infection cases caused by this fungus has gradually increased (4-6). However, this fungus is often difficult to distinguish from Aspergillus fumigatus in morphology, and most of these infections are associated with Aspergillus fumigatus in clinic. All of these presents a great challenge to the treatment of invasive fungal infections caused by A. lentulus $(7,8)$. At present, the pathogenesis and host immune response pathway of this fungus remains unclear.

The present study aims to determine the sensitivity of this fungus to commonly used antifungal agents through in vitro drug sensitivity, establish an infection model of $A$. lentulus infecting Galleria mellonella larvaes, and discuss the antifungal resistance, virulence and inflammatory factors' changes after the infection of larvae of $A$. lentulus separated from patients with chronic obstructive pulmonary disease (COPD) to reflect the host immune response.

\section{Methods}

\section{Strain source and groups}

\section{Strain source}

The A. lentulus standard strain: No. FH-5 (IFM54703, the gift from Chiba University, Japan). The clinical strain: Sputum specimens separated from one of COPD patients in our hospital. The study was approved by Medical ethics committee board of the First Affiliated Hospital of Xinjiang Medical University (No.: K201912-01) and informed consent was taken from all individual participants. The study was conducted in accordance with the Declaration of Helsinki (as revised in 2013). Steps for the isolation of strains in sputum specimens: In a biological safety cabinet, smear the sputum specimens on the surface of the PDA culture medium with a cotton swab and place them in a $37{ }^{\circ} \mathrm{C}$ incubator. After 3 days, the growth of colonies is observed. KOH direct microscopic examination showed thick, separated, bifurcated hyphae and conidia heads under the microscope. The bacterium was molecularly identified at Chiba University, Japan, and the $\beta$-tubulin gene (560 bp) gene was amplified. After blaste, the $\beta$-tubulin gene and A. lentulus had a homology of $99 \%$, and the phylogenetic tree also confirmed high homology. And registered in the Japanese gene database (DDBJ, http://www.ddbj.nig.ac.jp/ default.htm), the gene registration number is LC187284.

\section{Strain groups and culture}

Test grouping: A. lentulus standard strain (standard strain) group, A. lentulus strain isolated from a COPD patient (patient strain) group, and PBS (control) group. Young larvaes (purchased from Tianjin Huiyude Biological Technology Co., Ltd. Tinajin, China) with white color were selected, the selection criteria are 150-200 mg, no blackening and good mobility, then were refrigerated at $3-7^{\circ} \mathrm{C}$ for later use and incubated overnight at $37^{\circ} \mathrm{C}$ in the dark. The health index of plum blossom bacteria is listed in Table 1. Each group had 10 larvaes inoculated in a MEA 9-cm culture dish. After these were cultured up to the $9^{\text {th }}$ day at $25^{\circ} \mathrm{C}$, larvaes injection was performed with $A$. lentulus bacterial suspension at a concentration of $1 \times 10^{6} \mathrm{CFU}$. A. lentulus reached a steady state about 12 hours after infection with larvaes according to the observation data from the research group on the number of survivals after $A$. lentulus infection with larvaes, as shown in Figure 1. The culture was continued for 12 hours after the injection (9). Then, the infected larvae were rinsed with liquid nitrogen, and the supernatant collected. Take 10 samples for each group, A kit was used to detect the $(1,3)-\beta-D$-glucan (Megazyme, Shanghai, China) and galactomannan (Dana Biological Technology Co., Ltd., Tianjin, China) levels through enzyme-linked immunosorbent assay (ELISA). 


\section{In vitro chemosensitivity assay}

In vitro chemosensitivity assay was performed according to the CLSI M27-3A standard. The drug susceptibility test based on the micro-liquid-based dilution method, the specific operation is as follows: adjust the spore concentration of the tested strain to $1 \times 10^{6}-5 \times 10^{6} / \mathrm{mL}$ with a hemocytometer, and then dilute it with 1640 medium by 50 times. The tested drug concentration is diluted by multiples and then loaded in 96 micrometers. Orifice plate so that the final drug concentration of fluconazole in each well is $64,32,16,8,4,2,1,0.5,0.25$ and $0.125 \mu \mathrm{g} / \mathrm{mL}$, and the final drug concentration of the other antifungal drugs in

Table 1 The G. mellonella health index scoring system

\begin{tabular}{lcc}
\hline Category & Description & Description \\
\hline Activity & No movement & 0 \\
& Minimal movement on stimulation & 1 \\
& Move when stimulated & 2 \\
Melanisation & Move without stimulation & 3 \\
& Black larvae & 0 \\
& $\geq 3$ spots on beige larvae & 2 \\
$<3$ spots on beige larvae & 3 \\
& No melanisation & 4 \\
\hline
\end{tabular}

each well are respectively. For 16, 8, 4, 2, 1, 0.5, 0.25, 0.125, 0.0625 and $0.0312 \mu \mathrm{g} / \mathrm{mL}$, each drug is equipped with simple medium and bacterial solution control wells, and each drug sensitive plate is kept at $35^{\circ} \mathrm{C}$ Incubate statically for $48 \mathrm{~h}$ in a humidified incubator to interpret the results. Interpretation standard: The minimum inhibitory concentration (MIC) value of azole drugs is the minimum drug concentration that compares with the control $\geq 50 \%$ growth inhibition. Amphotericin B uses the minimum drug concentration of $100 \%$ growth inhibition compared with the control well as the drug MIC value. The lowest effective concentration MIC of Micafungi is defined as the lowest drug concentration when short and abnormally branched hyphae appear.

\section{Real-time polymerase chain reaction (PCR)}

The infected larvae in step 1 was fully ground with liquid nitrogen to extract the lymphoid blood cells. Total RNA was extracted with TRIzol Reagent (Invitrogen Co., USA) as described in its directions. The extracted RNA was reverse transcribed with 5X All-In-One RT MasterMix (with AccuRT Genomic DNA Removal Kit) (Invitrogen Co., USA). The expression of caspase- 1 and TNF- $\alpha$ was detected by real-time PCR used QuantiNova SYBR Green PCR Kit (Qiagen Co., Germany) and the first cDNA synthesis system is presented in Table 2, the fluorescence quantitative primers are presented in Table 3, and the reaction system and procedure are presented in Tables 4 and 5 .
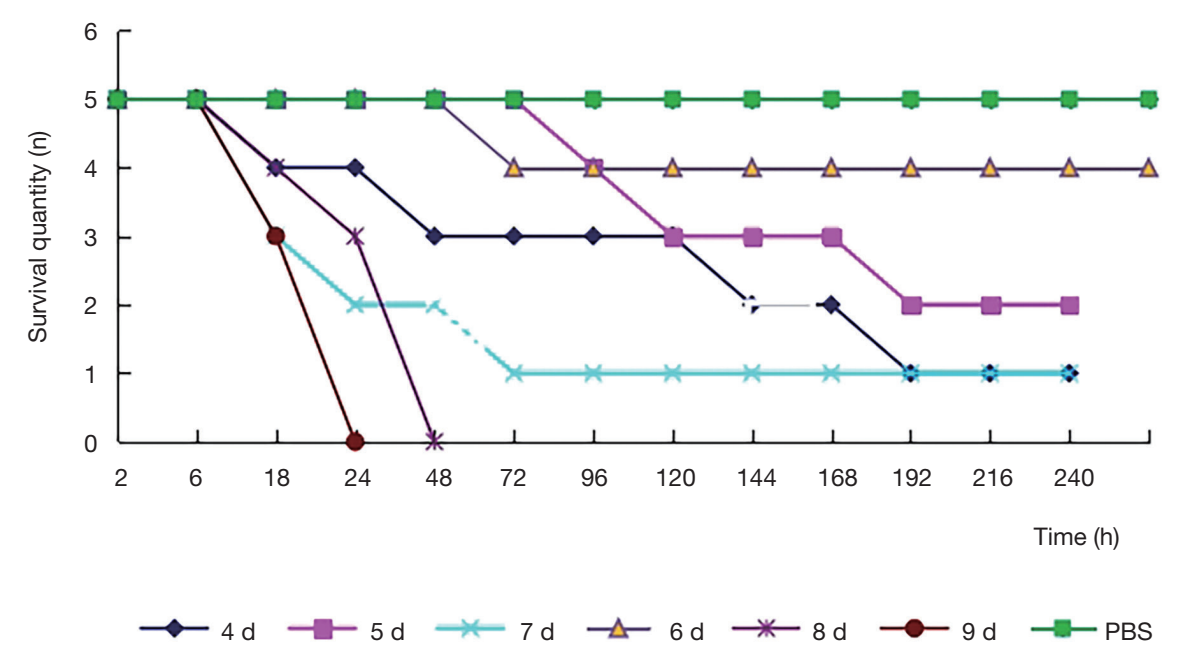

Figure 1 Survival number of $A$. lentulus patient group at a concentration of $1 \times 10^{6} \mathrm{CFU}$ after being infected with waxworm on different culture days. 
Table 2 The first cDNA synthesis system

\begin{tabular}{lc}
\hline Component & Volume \\
\hline Total RNA & Quantitative $800 \mathrm{ng}$ \\
Random primer $(0.1 \mu \mathrm{g} / \mu \mathrm{L})$ & $1 \mu \mathrm{L}$ \\
$2 \times$ TS reaction mix & $10 \mu \mathrm{L}$ \\
TransScript $^{\circledR} \mathrm{RT} / \mathrm{RI}$ Enzyme Mix & $1 \mu \mathrm{L}$ \\
gDNA remover & $1 \mu \mathrm{L}$ \\
RNase-free water & Up to $20 \mu \mathrm{L}$ \\
\hline
\end{tabular}

Table 3 The fluorescence quantitative primers

\begin{tabular}{lcc}
\hline Primer name & Sequence (5' to 3') & Primer size (bp) \\
\hline TNF- $\alpha$-F2 & AGGCCCACCTATCGTCAC & 109 \\
TNF- $\alpha-$ R2 & GCCAGGCTTTGTTTAGT & \\
Caspase-1-F2 & TTATTGATGGCGGTGCTA & 123 \\
Caspase-1-R2 & TTTCCCTGCTAATGTTGG & \\
Tubulin-F2 & CGTGGACTACGGCAAGAAGT & 128 \\
Tubulin-R2 & CACAGTCAGAGTGCTCCAGG & \\
\hline
\end{tabular}

Table 4 Fluorescence quantitative PCR system

\begin{tabular}{lc}
\hline Reagents & Volume $(\mu \mathrm{L})$ \\
\hline $2 \times$ SYBR green select mix & 5 \\
Forward primer & 0.7 \\
Reverse primer & 0.7 \\
ROX & 0.05 \\
cDNA & 1 \\
RNase-free water & Up to 10 \\
\hline
\end{tabular}

Table 5 Fluorescence quantitative PCR procedure

\begin{tabular}{lccc}
\hline Stage $(\mathrm{ABI})$ & Temperature $\left({ }^{\circ} \mathrm{C}\right)$ & Time & Circulate \\
\hline Pre degeneration & 95 & $2 \mathrm{~min}$ & 1 \\
Denaturation & 95 & $5 \mathrm{sec}$ & 40 \\
Annealing/extension & 60 & $30 \mathrm{sec}$ & \\
\hline
\end{tabular}

\section{Statistical analysis}

In the present study, SPSS 19.0 statistical software was used to process the data. The measurement data were expressed as mean \pm standard deviation. The enumeration data were expressed in percentage (\%). $W$-test was used for the normality test. $F$-test was used for the homogeneity test of variances. One-way analysis of variance (ANOVA) was used for comparisons among multiple groups, and least significant difference (LSD) was used for back testing. The nonparametric test was used to compare the mean of multiple samples that did not obey the normal distribution, or obeyed the normal distribution, but the variance was uneven. Counting data were analyzed using chi-square test. $\mathrm{P}<0.05$ was considered statistically significant.

\section{Results}

\section{Results of the in vitro drug sensitivity test}

The direct microscopic examination revealed that the separated hypanthium could be observed. After the culture, the colony morphology presented with a gray and white villous, and the conidia head could be observed under a microscope (Figure 2). After the molecular biological identification, the amplified $\beta$-tubulin gene (560 bp) and BLAST, and the homology between the $\beta$-tubulin gene and A. lentulus reached $99 \%$. Results of the in vitro drug sensitivity test: The MIC values of A. lentulus for amphotericin $\mathrm{B}, 5$-fluorouracil, fluconazole, itraconazole, voriconazole, miconazole and micafungin were 2, 64, $>64,0.5,1,4$ and $<0.015 \mu \mathrm{g} / \mathrm{mL}$, respectively. This suggests that $A$. lentulus has good sensitivity to itraconazole, voriconazole and micafungin (Table 6).

\section{The (1,3)- $\beta$-D-glucan level}

The results revealed that the concentration of galactomannan was $0.883 \pm 0.487 \mu \mathrm{g} / \mathrm{L}$ and the concentration of $(1,3)-\beta$-D-glucan was $0.037 \% \pm 0.029 \%$ in the control group, the concentration of galactomannan was $2.080 \pm$ $0.758 \mu \mathrm{g} / \mathrm{L}$ and the concentration of $(1,3)-\beta-\mathrm{D}$-glucan was $0.035 \% \pm 0.045 \%$ in the standard strain group, and the concentration of galactomannan was $1.635 \pm 0.193 \mu \mathrm{g} / \mathrm{L}$ and the concentration of $(1,3)-\beta-D$ - glucan was $0.033 \% \pm 0.029 \%$ in the patient group. There was no difference in $(1,3)-\beta-\mathrm{D}-$ glucan concentration between two groups and the control group, suggesting that this may not be involved in the formation of the virulence of early pathogenic fungi. For these two groups, the concentration of galactomannan was higher, when compared to the control group, and the difference was statistically significant. The lack of difference between these two groups (Figure 3) further confirm that 

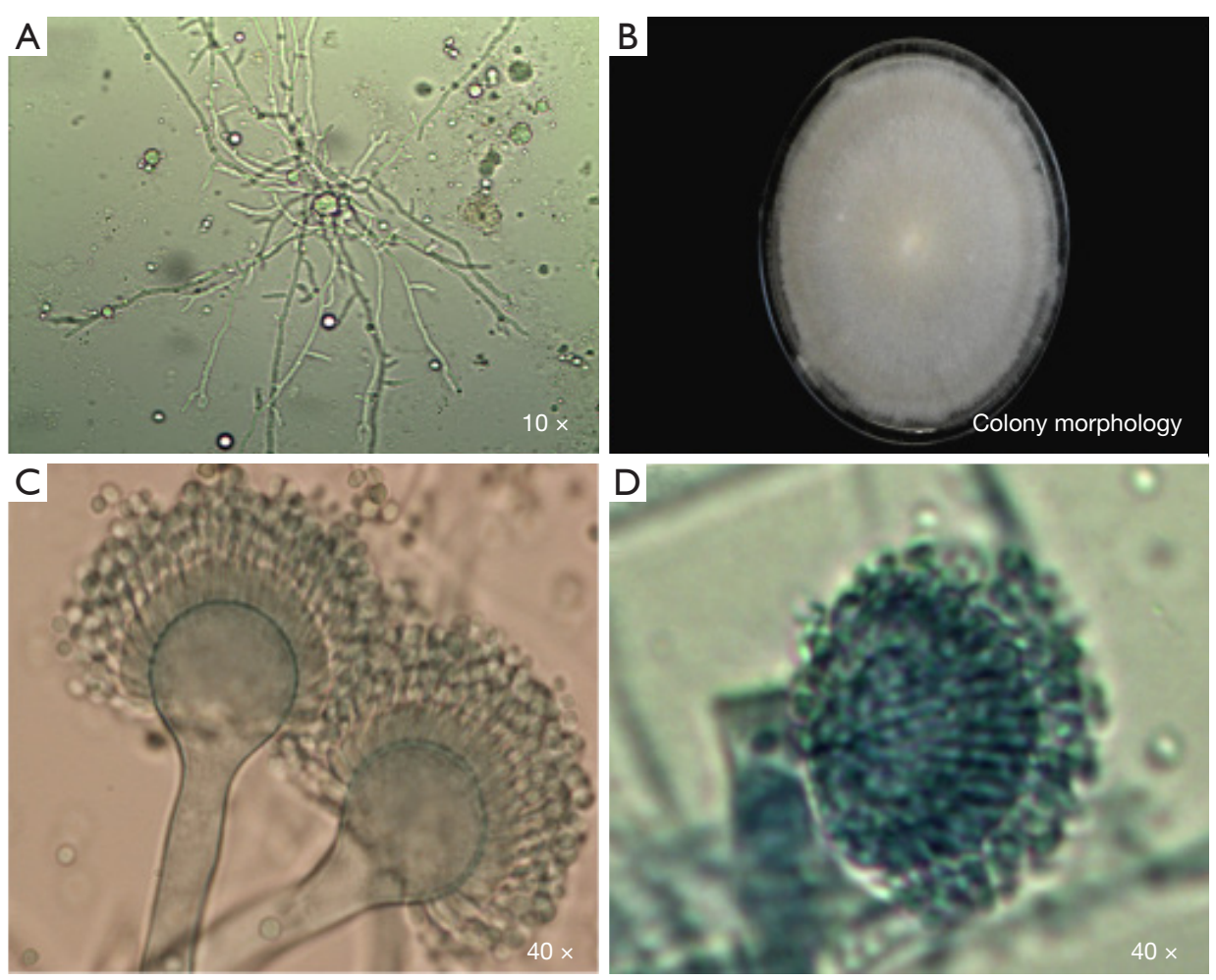

Figure 2 The morphological identification of Aspergillus lentulus. (A) The hyphal separation of branches in the sputum was observed by $\mathrm{KOH}$ direct microscopic examination. (B) The colony was cultured at $25^{\circ} \mathrm{C}$ for 14 days, and presented with a gray and white villous shape. (C,D) The Aspergillus lentulus conidial head is shown.

Table 6 In vitro antifungal drug sensitivity of $A$. lentulus

\begin{tabular}{lccccccc}
\hline \multirow{2}{*}{ Strains } & \multicolumn{5}{c}{$\mathrm{MIC}_{90}(\mathrm{\mu g} / \mathrm{mL})$} \\
\cline { 2 - 7 } & AMPH & $5-\mathrm{FC}$ & FLCZ & ITCZ & VRCZ & MCZ & MCFG \\
\hline A. lentulus 2429 & 2 & 64 & $>64$ & 0.5 & 1 & 4 & $\leq 0.015$ \\
A. lentulus FH5 & 2 & 64 & $>64$ & 0.5 & 1 & 2 & $\leq 0.03$ \\
\hline
\end{tabular}

MIC, minimum inhibitory concentration; AMPH, amphotericin B; 5-FC, 5-fluorouracil; FLCZ, fluconazole; ITCZ, itraconazole; VRCZ, voriconazole; MCZ, miconazole; MCFG, micafungin.

this significantly increased as a fungal virulence factor at the beginning of the infection.

\section{TNF- $\alpha$ and caspase-1 expression levels}

These results revealed that the expression level of TNF- $\alpha$ and caspase- 1 in the control group was $1.040 \pm 0.303$ and $1.083 \pm 0.400$, respectively, the expression level of TNF- $\alpha$ and caspase- 1 in the standard strain group was $1.946 \pm 0.845$ and $2.696 \pm 1.729$, respectively, and the expression level of TNF- $\alpha$ and caspase- 1 in the patient strain group was $2.486 \pm 1.114$ and $4.612 \pm 1.746$, respectively. Furthermore, the levels of TNF- $\alpha$ and caspase- 1 in the larvae after infection were higher in these two groups than in the control group $(\mathrm{P}<0.05)$. This suggests that the induced host immune response was accompanied by the participation of the caspase- 1 pathway, and TNF- $\alpha$ was involved in the host immune response (Figure 4). 

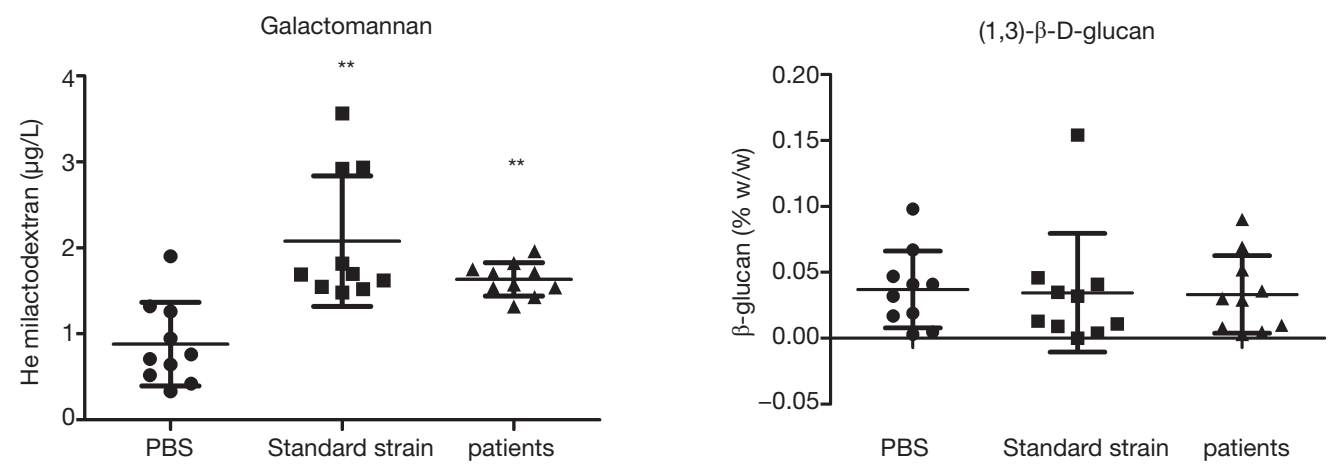

Figure 3 The horizontal mapping of two factors in the infected Galleria mellonella. ${ }^{* *}, \mathrm{P}<0.01$.
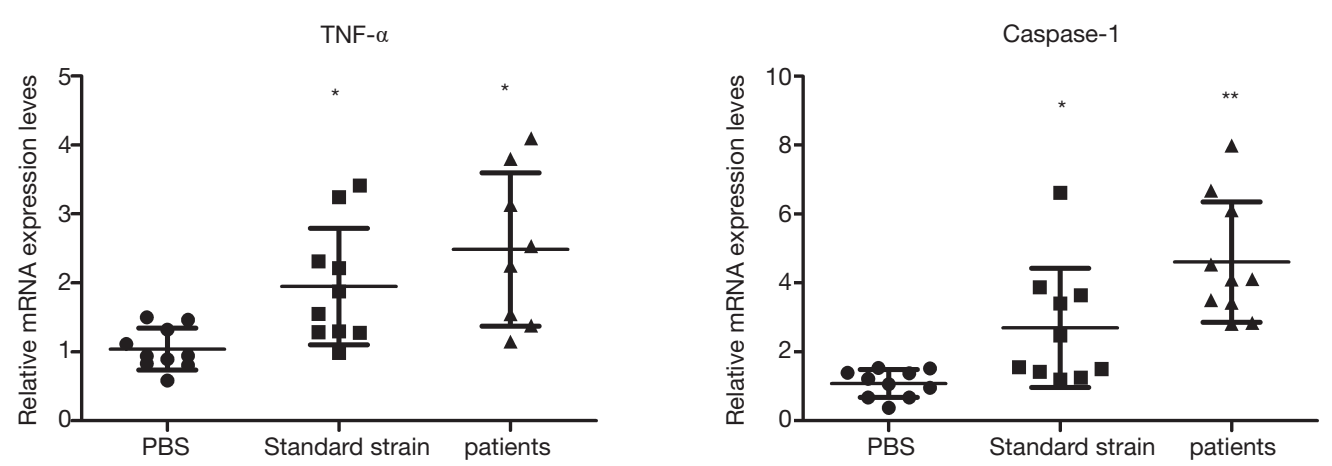

Figure 4 The mRNA expression level of two genes in the infected Galleria mellonella. *, $\mathrm{P}<0.05$; **, $\mathrm{P}<0.01$.

\section{Discussion}

The results of the present study revealed that $A$. lentulus has good sensitivity to itraconazole, voriconazole and micafungin. However, $(1,3)-\beta$-D-glucan was negative in the two groups. The level of galactomannan in the two groups was higher than that in the control group, showing that there was statistical significance, but there was no statistical difference between the standard strain group and patient strain group. After the infection of larvae, those in the control group and standard strain group induce the increase in caspase- 1 and TNF- $\alpha$ in Galleria mellonella larvae. These elevated levels were statistically significant in both groups, but there was no significant difference between the two groups.

Fungal virulence is influenced by a variety of factors, and some of which directly lead to host disease. Certain key virulence factors directly lead to host disease. Fungal morphology changes and fungal surface antigens ( $\beta$-glucan, chitin, mannan, etc.) are important components for activating the host innate immune system. The (1,3)- $\beta-\mathrm{D}-$ glucan and galactomannan are not only important virulence factors to induce innate immunity, but also clinical diagnostic indexes for early invasive aspergillus infection. When the body is invaded by fungi, it initiates the surface pattern recognition receptor of the innate immune system or the induction system, in order to activate the inflammatory response, and promote the release of inflammatory factors, such as IL- $1 \beta$, TNF- $\alpha$ and IL-18. At present, as an important component of the natural immune system, which is highly dependent on the recognition and response of pathogenic microorganisms, NLRP3 inflammasome plays an important role in the pathogenesis of fungal virulence and host immune response. In the present study, the virulence of $A$. lentulus and the level of host immune inflammatory factors were measured for the first time, and the possible host immune response mechanism was discussed.

The prognosis of fungal infection was correlated to its own virulence and antifungal drug sensitivity. At present, aspergillus fumigatus has resistance to itraconazole, 
voriconazole and posaconazole at $0.2-2.6 \%, 0.8-3.1 \%$ and $0.2-2.2 \%$, respectively. However, there is no report on the overall drug resistance rate for A. lentulus, which is a sister fungus of aspergillus fumigatus. As reported, the MIC values of $A$. lentulus for itraconazole, voriconazole and posaconazole were $0.12-16,0.25-16$ and $0.03-2 \mathrm{mg} / \mathrm{L}$, respectively $(7,8)$. More strains of high-MIC value of azole medicine are present, which greatly increases the difficulty of the invasive infection treatment caused by the fungus. The present experimental strain was the first $A$. lentulus isolated in China, and the MIC values to amphotericin B, 5-fluorouracil, fluconazole, itraconazole, voriconazole, miconazole and micafungin were 2, 64, >64, 0.5, 1, 4 and $<0.015 \mu \mathrm{g} / \mathrm{mL}$, respectively. This suggests that $A$. lentulus has good sensitivity to itraconazole, voriconazole and micafungin, and that the in vitro drug sensitivity is basically consistent with the control standard strain.

The $(1,3)-\beta$-D-glucan is a common structural component of all fungal cell walls, while galactomannan is the main skeleton component of aspergillus cell walls and an important immunogenic factor, which can induce phagocytosis and the release of inflammatory factors through receptors, such as dectin-1, on the surface of host cells. At present, the detection of these above two indexes has been used as an index for the early diagnosis of systemic Aspergillus infection, which has high sensitivity and specificity. In the present study, $(1,3)-\beta-D$-glucan did not increase in the two groups of larvaes with $A$. lentulus infection, while galactomannan increased, and the difference was statistically significant. These results suggest that cell wall galactomannan, as a virulence factor, is involved in the host immune response during the immune response of larvae infected with $A$. lentulus, and increases at 12 hours after infection. This can be used as a monitoring index for early $A$. lentulus infection. Some studies have revealed that compared with the dendritic rapid growth of Aspergillus fumigatus in tissue, A. lentulus is wrapped in the form of nodules by immune cells in the early stage of infection. Hence, the immune occurrence of $A$. lentulus relatively lags behind (10). The nodes in the present study were detected at 12 hours after larvae infection. Therefore, it was speculated that $(1,3)-\beta$-D-glucan could not be detected due to this factor. In summary, it was considered that galactomannan has a greater advantage as an indicator for monitoring early invasive $A$. lentulus infection.

Inflammasome is a kind of polyprotein complex in cells. At present, all known inflammasomes can activate pro-caspase- 1 into caspase- 1 through their own specific pathways, and induce the secretion of inflammatory cytokines, such as IL-1 $\beta$, TNF- $\alpha$ and IL- 18 . The presently known inflammasomes include NLRP inflammasomes, AIM2 inflammasomes, and NLRP6 inflammasomes, which are presently the most complex NLRP3 inflammasomes (11-13). Previous studies have shown that NLRP3 inflammasomes can also be directly or indirectly activated by some fungal or fungal components, and play an important role in immune function (14-17). The study conducted by Saïd-Sadier et al. confirmed that Aspergillus fumigatus can activate the NLRP3 inflammasome, and induce an IL-1 $\beta$ mediated inflammatory response, when Aspergillus fumigatus is exposed to $\beta$-glucan in the hyphal state (18). Based on this, it was speculated that NLRP3 inflammasomes are involved in the host immune response of $A$. lentulus. This above hypothesis was confirmed by the significant increase in caspase- 1 and inflammatory factor TNF- $\alpha$ in the two groups of larvae after infection. However, the NLRP3mediated immune response is complex $(19,20)$, and the specific mechanism needs to be further investigated.

In the present study, the standard strain and A. lentulus strain isolated for the first time in China exhibited a consistency in drug sensitivity in vitro, the virulence factor monitoring index, and the inflammatory factor level of host immune response, suggesting that there is no significant regional difference in the virulence factor and host immune response induced by $A$. lentulus.

\section{Conclusions}

There was no significant difference in virulence factor and host inflammatory response between the $A$. lentulus isolated from COPD patients and standard strains. Galactomannan has more advantages in the early detection of $A$. lentulus invasive infection. The caspase-1-mediated inflammasome pathway may be involved in the host immune response to $A$. lentulus.

\section{Acknowledgments}

Funding: Project supported by the National Natural Science Foundation of China (grant No. 81760360).

\section{Footnote}

Data Sharing Statement: Available at http://dx.doi. 
org/10.21037/jtd-20-961

Conflicts of Interest: All authors have completed the ICMJE uniform disclosure form (available at http://dx.doi. org/10.21037/jtd-20-961). All authors have no conflicts of interest to declare.

Ethical Statement: The authors are accountable for all aspects of the work in ensuring that questions related to the accuracy or integrity of any part of the work are appropriately investigated and resolved. The study was conducted in accordance with the Declaration of Helsinki (as revised in 2013). The study was approved by Medical ethics committee board of the First Affiliated Hospital of Xinjiang Medical University (No.: K201912-01) and informed consent was taken from all individual participants.

Open Access Statement: This is an Open Access article distributed in accordance with the Creative Commons Attribution-NonCommercial-NoDerivs 4.0 International License (CC BY-NC-ND 4.0), which permits the noncommercial replication and distribution of the article with the strict proviso that no changes or edits are made and the original work is properly cited (including links to both the formal publication through the relevant DOI and the license). See: https://creativecommons.org/licenses/by-nc-nd/4.0/.

\section{References}

1. Le Mauff F, Bamford NC, Alnabelseya N, et al. Molecular mechanism of Aspergillus fumigatus biofilm disruption by fungal and bacterial glycoside hydrolases. J Biol Chem 2019;294:10760-72.

2. Balajee SA, Gribskov JL, Hanley E, Aspergillus lentulus sp. nov., a new sibling species of A. fumigatus. Eukaryot Cell 2005;4:625-32.

3. Alhambra A, Catalán $M$, Moragues MD, et al. Isolation of Aspergillus lentulus in Spain from a critically ill patient with chronic obstructive pulmonary disease. Rev Iberoam Micol 2008;25:246-9.

4. Zbinden A, Imhof A, Wilhelm MJ, et al. Fatal outcome after heart transplantation caused by Aspergillus lentulus. Transpl Infect Dis 2012;14:E60-3.

5. Montenegro G, Sánchez Puch S, Jewtuchowicz VM, et al. Phenotypic and genotypic characterization of Aspergillus lentulus and Aspergillus fumigatus isolates in a patient with probable invasive aspergillosis. J Med Microbiol
2009;58:391-5.

6. Symoens F, Haase G, Pihet M, et al. Unusual Aspergillus species in patients with cystic fibrosis. Med Mycol 2010;48 Suppl 1:S10-6.

7. Alcazar-Fuoli L, Mellado E, Alastruey-Izquierdo A, et al. Aspergillus section Fumigati: antifungal susceptibility patterns and sequence-based identification. Antimicrob Agents Chemother 2008;52:1244-51.

8. Alastruey-Izquierdo A, Alcazar-Fuoli L, CuencaEstrella M. Antifungal susceptibility profile of cryptic species of Aspergillus published correction appears in Mycopathologia 2015 Apr;179(3-4):333-4. Mycopathologia 2014;178:427-33.

9. Dai JJ, Zhou WB, Li HL, et al. Preliminary study on the autophagy mechanism of artesunate against Aspergillus fumigatus infection. Chinese Journal of Microecology 2018;30:287-90.

10. Alcazar-Fuoli L, Buitrago M, Gomez-Lopez A, et al. An alternative host model of a mixed fungal infection by azole susceptible and resistant Aspergillus spp strains. Virulence 2015;6:376-84.

11. Schroder K, Tschopp J. The inflammasomes. Cell 2010;140:821-32.

12. Gross O, Poeck H, Bscheider M, et al. Syk kinase signalling couples to the Nlrp3 inflammasome for antifungal host defense. Nature 2009;459:433-6.

13. Hise AG, Tomalka J, Ganesan S, et al. An essential role for the NLRP3 inflammasome in host defense against the human fungal pathogen Candida albicans. Cell Host Microbe 2009;5:487-97.

14. Kankkunen P, Teirilä L, Rintahaka J, et al. (1,3)-betaglucans activate both dectin-1 and NLRP3 inflammasome in human macrophages. J Immunol 2010;184:6335-42.

15. Kumar H, Kumagai $Y$, Tsuchida T, et al. Involvement of the NLRP3 inflammasome in innate and humoral adaptive immune responses to fungal beta-glucan. J Immunol 2009;183:8061-7.

16. Shen HH, Yang YX, Meng X, et al. NLRP3: A promising therapeutic target for autoimmune diseases. Autoimmun Rev 2018;17:694-702.

17. Alhallaf R, Agha Z, Miller CM, et al. The NLRP3 Inflammasome Suppresses Protective Immunity to Gastrointestinal Helminth Infection. Cell Rep 2018;23:1085-98.

18. Saïd-Sadier N, Padilla E, Langsley G, et al. Aspergillus fumigatus stimulates the NLRP3 inflammasome through a pathway requiring ROS production and the Syk tyrosine 
kinase. PLoS One 2010;5:e10008.

19. Quan JH, Huang R, Wang Z, et al. P2X7 receptor mediates NLRP3-dependent IL-1 $\beta$ secretion and parasite proliferation in Toxoplasma gondii-infected human small intestinal epithelial cells. Parasit Vectors 2018;11:1.

Cite this article as: Zhang LJ, Wang XD, Ji MS, Hasimu H, Abliz P. Characterisation of a clinical isolated Aspergillus lentulus strain using a Galleria mellonella infection model. J Thorac Dis 2021;13(2):803-811. doi: 10.21037/jtd-20-961
20. Kang MJ, Jo SG, Kim DJ, et al. NLRP3 inflammasome mediates interleukin-1 $\beta$ production in immune cells in response to Acinetobacter baumannii and contributes to pulmonary inflammation in mice. Immunology 2017;150:495-505. 